\title{
Multi-quasi-parabolic ionosphere model with EF-valley
}

\author{
Jie Sun ${ }^{1,2}$, Xiao-Juan Zhang, ${ }^{1}{ }^{\star}$, Shun Wang ${ }^{1}$, Zhao-Qian Gong ${ }^{1}$, Guang-You Fang ${ }^{1}$ \\ ${ }^{1}$ Key Laboratory of Electromagnetic Radiation and Sensing Technology, Chinese Academy of Sciences, Beijing, China \\ ${ }^{2}$ University of Chinese Academy of Sciences, Beijing, China
}

\author{
Article history \\ Received April 29, 2015; accepted January 12, 2016. \\ Subject classification: \\ Wave propagation, Ionosphere model, EF-valley, Ray-tracing, Oblique sounding ionogram.
}

\begin{abstract}
We improve the conventional multi-quasi-parabolic (MQP) model and develop two MQP models with EF-valley by applying the predicted EF-valley parameter of the newest version of the International Reference Ionosphere. On the basis of the conventional MQP model and these two improved MQP models with EF-valley, we generate one-hop oblique sounding ionograms between Wuhan and Beijing. Comparisons between the generated ionograms and the sounding ionogram in the experiments illustrate that the improved MQP models are more suitable for describing the true ionosphere than the conventional MQP model is. In contrast to the conventional MQP model, the improved MQP models can increase the accuracy of ray tracing and reduce the errors in shortwave single location and over-the-horizon-radar registration.
\end{abstract}

\section{Introduction}

Radio propagation depends uniquely on ionospheric electron density. Research on radio propagation usually refers to the mathematical description of ionospheric electron concentration as the ionosphere model. This model significantly affects the prediction and evaluation of shortwave communication, and the accuracy of this model determines the positioning precision of over-the-horizon-radar (OTHR) and satellite navigation [Li (W.M.) et al. 2012]. The International Reference Ionosphere (IRI) is the most frequently used ionosphere model in qualitative analysis and is an empirical standard model. The major data sources of IRI are the worldwide network of ionosondes, and the newest version of this model is labelled IRI2012. IRI is a statistical prediction model; thus, the provided parameters differ from the real-time data. A common ionosphere model in engineering application (particularly in the field of OTHR) is the multi-quasi-parabolic (MQP) model because this model can represent the real-time ionosphere when the parameters of the various layers are updated in a timely manner. The MQP model describes different ionosphere layers with several quasi-parabolic segments, and the linking segment among layers is a counter-parabolic function. After Dyson and Bennett [1988] introduced the MQP model, this model has been used in various engineering fields. Bourgeois et al. [2005, 2006] applied the MQP model for target tracking in OTHR. Baker and Lambert [1989] and Wang et al. [2009] estimated ranges for high-frequency single station location with this model. Gasse et al. [1999] estimated mean bearing derivations with the MQP model. Hou et al. [2015] employed the dynamic MQP model to compute sky-wave radar detection performance. Li (X.D.) et al. [2012] developed a signal model by utilizing the MQP ionospheric model to derive an optimum detector for MIMO-OTHR. Liu (R.Y.) et al. [2008] applied the MQP model to shortwave ray tracing in the ionosphere. Wang et al. [2010] presented an F1 layer inversion method based on MQP model.

The data obtained from the incoherent scatter radar indicate the presence of a valley between the $\mathrm{E}$ and $\mathrm{F}$ layers; the depth and width of the valley are significantly less in daytime than at night [Mahajan et al. 1990], but ionosonde could not sound that region. No incoherent scatter radar has been developed in China; therefore, the MQP model used at present does not include an EFvalley. This lack reduces the accuracy of systems which use the ionosphere model. The depth of the valley from IRI2012 is a relative value, and the corresponding width is an absolute value; the former is closer to the actual situation than the latter is. The current work combines the predicted values of the EF-valley from IRI2012 with the other QP layer parameters derived from vertical sounding to generate two improved MQP models with EF-valley, namely, the MQP model which uses the depth of valley (MQP-VD) and the MQP model which uses the depth and width of valley (MQP-VDW). Oblique sounding ionograms demonstrate the group delay dependence over a defined frequency range; this group 


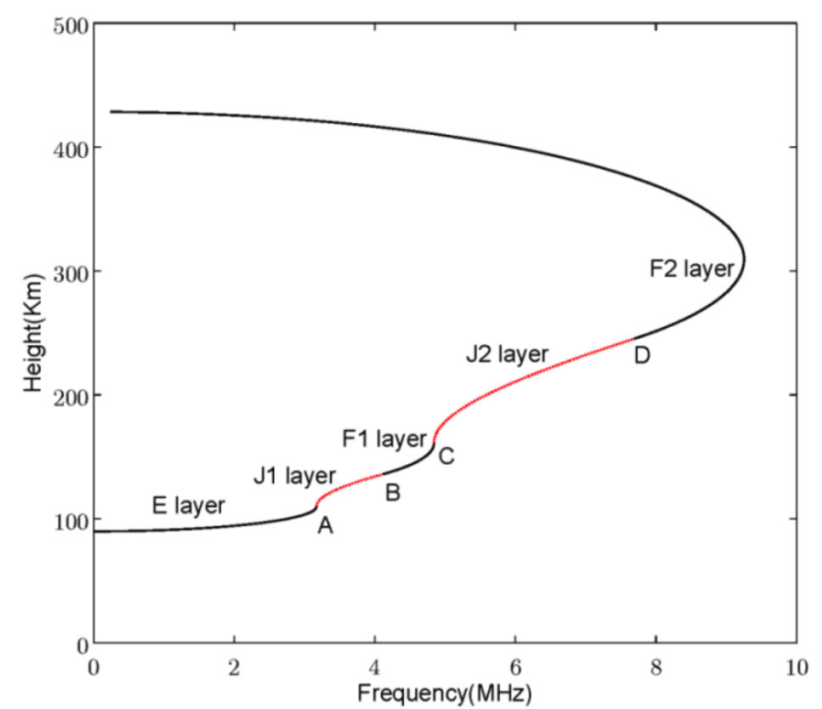

Figure 1. MQP ionosphere model.

delay function depends upon the ionospheric structure in a significantly more complex manner than it does for vertical sounding [Krasheninnikov et al. 1996]. Thus, we generate one-hop oblique sounding ionograms between Wuhan and Beijing with the two improved MQP models and a conventional MQP model. We also compare the three oblique sounding ionograms with the experimental ionogram from the ionosonde. The results illustrate that in contrast to the conventional MQP model, the improved MQP models effectively increase the accuracy of ray tracing and reduce the errors in shortwave single location and OTHR registration.

\section{Conventional MQP model}

The QP model describes the profile of electron concentration in the ionosphere with a quasi-parabolic segment. A relationship exists between plasma frequency and electron concentration $\left(f_{N}^{2}=80.8 N_{e}\right)$; therefore, the QP model for a single layer is often determined with Equations (1a) and (1b) [Dyson and Bennett 1988].

$$
\left\{\begin{array}{cc}
f_{N}^{2}=a-b\left(1-\frac{r_{m}}{r}\right)^{2} & r_{b}<r<r_{t} \\
0 & \text { otherwise }
\end{array}\right.
$$

where $r$ is the radial distance from the Earth's center, $r_{b}$ is the value of $r$ at the layer base, $y_{m}$ is the layer semithickness, $r_{m}=r_{b}+y_{m}$ is the value of $r$ where the plasma frequency reaches maximum, $a=f_{0}^{2}\left(f_{0}\right.$ is the critical frequency of the layer), $b$ is given by Equation (2a) and $r_{t}$ is given by Equation ( $\left.2 \mathrm{~b}\right)$.

$$
\left\{\begin{array}{l}
b=a\left(\frac{r_{b}}{y_{m}}\right)^{2} \\
r_{t}=\frac{r_{m} r_{b}}{r_{b}-y_{m}}
\end{array}\right.
$$

The MQP model is developed in this manner (see Figure 1). The $\mathrm{E}$ and $\mathrm{F} 1$ layers are joined by the $\mathrm{J} 1$ joining layer, and the joining points are points $\mathrm{A}$ and $\mathrm{B}$. The $\mathrm{F} 1$ and $\mathrm{F} 2$ layers are joined by the J2 joining layer, and the joining points are points $\mathrm{C}$ and $\mathrm{D}$. The equations which describe the MQP ionosphere model can be written as Equations (3a)-(3e); the parameters in these equations are similar to those in Equation (1). The joining layers are described by the counter-QP function, as mentioned above; therefore, $r_{m J 1}$ and $r_{m J 2}$ represent the minimum radial distance of the J1 and J2 layers, respectively.

$$
\begin{aligned}
& \left\{\begin{array}{l}
f_{\mathrm{NE}}^{2}=a_{\mathrm{E}}-b_{\mathrm{E}}\left(1-\frac{r_{m \mathrm{E}}}{r}\right) \star \\
f_{\mathrm{N} 11}^{2}=a_{\mathrm{J} 1}-b_{\mathrm{J} 1}\left(1-\frac{r_{m \mathrm{j} 1}}{r}\right) \star \star \\
f_{\mathrm{NF} 1}^{2}=a_{\mathrm{F} 1}-b_{\mathrm{F} 1}\left(1-\frac{r_{m \mathrm{~F} 1}}{r}\right) \star \star \star \\
f_{\mathrm{N} 22}^{2}=a_{\mathrm{J} 2}-b_{\mathrm{J} 2}\left(1-\frac{r_{m \mathrm{j} 2}}{r}\right) \star \star \star \star \\
f_{\mathrm{NF} 2}^{2}=a_{\mathrm{F} 2}-b_{\mathrm{F} 2}\left(1-\frac{r_{m \mathrm{~F} 2}}{r}\right) \star \star \star \star \star
\end{array}\right. \\
& \text { * } \quad \text { E layer }\left(r_{b} \leq r<r_{\mathrm{A}}\right) \\
& \star \star \quad \text { J1 joining layer }\left(r_{\mathrm{A}} \leq r<r_{\mathrm{B}}\right) \\
& \star \star \star \quad \text { F1 layer }\left(r_{\mathrm{B}} \leq r<r_{\mathrm{C}}\right) \\
& \star \star \star \star \quad \text { J2 joining layer }\left(r_{\mathrm{C}} \leq r<r_{\mathrm{D}}\right) \\
& \star \star \star \star \star \text { F2 layer }\left(r_{\mathrm{D}} \leq r<r_{\text {tF2 }}\right)
\end{aligned}
$$

The parameters of the joining layers and the values of the joining points are determined as follows:

Firstly, we assume that the J1 and E layers join at the peak of the latter. Then $a_{\mathrm{J} 1}=a_{\mathrm{E}}, r_{m \mathrm{~J} 1}=r_{m \mathrm{E}}$. The J2 and $\mathrm{F} 1$ layers converge at the peak of the latter; subsequently, $a_{\mathrm{J} 2}=a_{\mathrm{F} 1}, r_{m \mathrm{~J} 2}=r_{m \mathrm{~F} 1} r_{t \mathrm{tF} 2}$ is the $r_{t}$ value of the F2 layer.

Secondly, the parameter of the J1 layer $b_{\mathrm{J} 1}$ and the height of joining point $\mathrm{B}$ are computed by matching the plasma frequency and its gradient according to Equations (4a) and (4b). Then, the answers are given by Equations (5a) and (5b).

$$
\begin{aligned}
& \left\{\begin{array}{c}
\left.f_{\mathrm{NF} 1}^{2}\right|_{r=r_{\mathrm{B}}}=\left.f_{\mathrm{N} 11}^{2}\right|_{r=r_{\mathrm{B}}} \\
\left.\frac{\partial f_{\mathrm{NF} 1}^{2}}{\partial r}\right|_{r=r_{\mathrm{B}}}=\left.\frac{\partial f_{\mathrm{N} 1}^{2}}{\partial r}\right|_{r=r_{\mathrm{B}}}
\end{array}\right. \\
& \left\{\begin{array}{l}
r_{\mathrm{B}}=\frac{r_{\mathrm{F} 1} b_{\mathrm{F} 1}\left(r_{\mathrm{F} 1} / r_{\mathrm{E}}-1\right)}{a_{\mathrm{F} 1}-a_{\mathrm{E}}+b_{\mathrm{F} 1}\left(r_{\mathrm{F} 1} / r_{\mathrm{E}}-1\right)} \\
b_{\mathrm{J} 1}=\frac{-r_{\mathrm{H} 1} b_{\mathrm{F} 1}\left(1-r_{\mathrm{H} 1} / r_{\mathrm{B}}\right)}{r_{\mathrm{E}}\left(1-r_{\mathrm{E}} / r_{\mathrm{B}}\right)}
\end{array}\right.
\end{aligned}
$$


Finally, the parameter $b_{\mathrm{J} 2}$ of the $\mathrm{J} 2$ layer and the height $r_{\mathrm{D}}$ of joining point $\mathrm{D}$ are computed as described above. The values are represented by Equations (6a) and (6b)

$$
\left\{\begin{array}{l}
r_{\mathrm{D}}=\frac{r_{\mathrm{H} 2} b_{\mathrm{F} 2}\left(r_{\mathrm{H} 2} / r_{\mathrm{H} 1}-1\right)}{a_{\mathrm{F} 2}-a_{\mathrm{F} 1}+b_{\mathrm{F} 2}\left(r_{\mathrm{F} 2} / r_{\mathrm{F} 1}-1\right)} \\
b_{\mathrm{J} 2}=\frac{-r_{\mathrm{H} 2} b_{\mathrm{F} 2}\left(1-r_{\mathrm{H} 2} / r_{\mathrm{D}}\right)}{r_{\mathrm{H} 1}\left(1-r_{\mathrm{H} 1} / r_{\mathrm{D}}\right)}
\end{array}\right.
$$

\section{Improved MQP model}

The vertical sounding ionosonde cannot obtain information with regard to the EF-valley. In this study, the parameters of this factor are obtained from IRI2012. The real-time data from vertical sounding ionosonde are combined with the predicted data from IRI2012 to develop the improved MQP models with EF-valley. The joining parameters of the EF-valley with the $\mathrm{E}$ and $\mathrm{F}$ layers are computed as follows.

\section{A. MQP-VD model}

The relative depth " $m$ " value of the EF-valley can be obtained based on IRI2012. The value of $a_{\mathrm{V}}$ is computed by $a_{\mathrm{V}}=\mathrm{m} \cdot a_{\mathrm{E}}$. The heights $r_{\mathrm{A}}$ and $r_{\mathrm{B}}$ of joining points $\mathrm{A}$ and $\mathrm{B}$ are given by Equations (7a) and (7b). The EF-valley of MQP-VD is denoted in Figure 2 by a solid line. The functions which describe the MQP-VD model are represented by Equations (8a)-(8c) .

$$
\begin{aligned}
& \left\{\begin{array}{l}
r_{\mathrm{A}}=\frac{r_{\mathrm{E}}}{1-\sqrt{\left(a_{\mathrm{E}}-a_{\mathrm{V}}\right) / b_{\mathrm{E}}}} \\
r_{\mathrm{B}}=\frac{r_{\mathrm{F}}}{1-\sqrt{\left(a_{\mathrm{F}}-a_{\mathrm{V}}\right) / b_{\mathrm{F}}}}
\end{array}\right. \\
& \begin{cases}f_{\mathrm{NE}}^{2}=a_{\mathrm{E}}-b_{\mathrm{E}}\left(1-\frac{r_{m \mathrm{E}}}{r}\right) & \left(r_{b} \leq r \leq r_{\mathrm{A}}\right) \\
f_{\mathrm{V}}^{2}=a_{\mathrm{V}} & \left(r_{\mathrm{A}}<r<r_{\mathrm{B}}\right) \\
f_{\mathrm{NF} 2}^{2}=a_{\mathrm{F} 2}-b_{\mathrm{F} 2}\left(1-\frac{r_{m \mathrm{~F} 2}}{r}\right) & \left(r_{\mathrm{B}} \leq r<r_{\mathrm{tF} 2}\right)\end{cases}
\end{aligned}
$$

\section{B. MQP-VDW model}

Both the relative depth " $\mathrm{m}$ " and absolute width "W" of the EF-Valley obtained from IRI2012 are used in the MQP-VDW model. The parameters of the joining layer are computed according to the values of $a_{\mathrm{V}}$ and $\mathrm{W}$, and the joining segment should pass through point $\left(a_{\mathrm{E}}, r_{\mathrm{Q}}\right)$, (where $r_{\mathrm{Q}}=r_{\mathrm{E}}+\mathrm{W}$ ) and converge with the straight line at the bottom of the valley, i.e., $a_{\mathrm{J}}=a_{\mathrm{V}}$. The joining layer intersects with the $\mathrm{F} 2$ layer at point $\mathrm{M}$ (see Figure 2). The gradients of this layer are continuous at point M. We obtain Equation (9) according to the aforementioned rules, where $r_{\mathrm{M}}, b_{\mathrm{J}}$ and $r_{m \mathrm{~J}}$ are unknown pa-

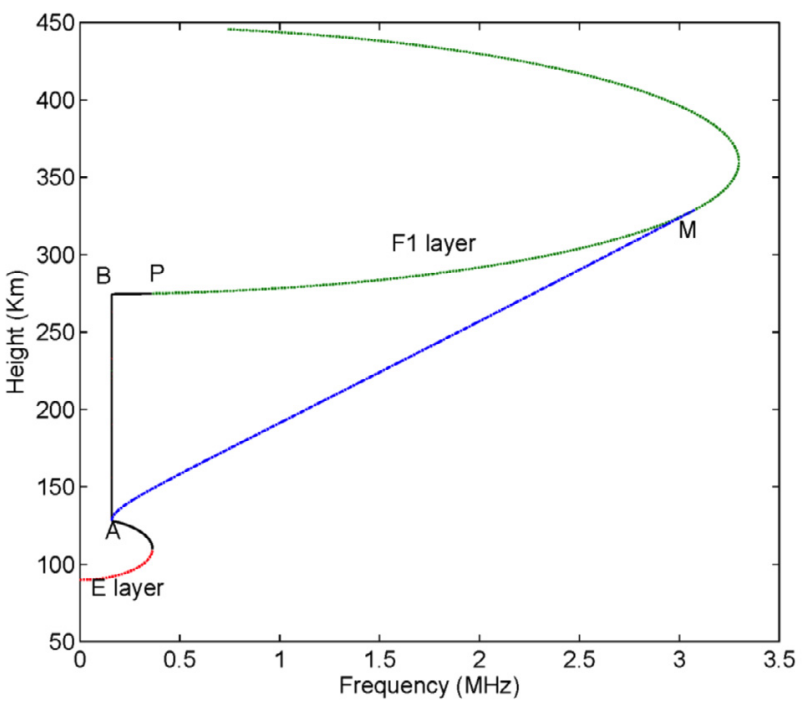

Figure 2. MQP-VD ionosphere model.

rameters. The valley of MQP-VDW is highlighted by the dotted and dashed line in Figure 2. If the computed $r_{m \mathrm{~J}}$ value is smaller than $r_{\mathrm{A}}$, which reduces the valley depth, then only the first two formulas in Equations (9a)-(9c) are used to compute $r_{\mathrm{M}}$ and $b_{\mathrm{J}}$. This procedure maintains a satisfactory valley depth (i.e., $r_{m J}=r_{\mathrm{A}}$ ). The values of $r_{\mathrm{M}}$ and $b_{\mathrm{J}}$ are given by Equations (10a) and (10b).

$$
\begin{aligned}
& \left\{\begin{array}{l}
\left.f_{\mathrm{NJ}}^{2}\right|_{r=r_{\mathrm{M}}}=\left.f_{\mathrm{NF} 2}^{2}\right|_{r=r_{\mathrm{M}}} \\
\left.\frac{\partial f_{\mathrm{NJ}}^{2}}{\partial r}\right|_{r=r_{\mathrm{M}}}=\left.\frac{\partial f_{\mathrm{NF} 2}^{2}}{\partial r}\right|_{r=r_{\mathrm{M}}} \\
f_{\mathrm{NJ}}^{2}=a_{\mathrm{V}}+b_{\mathrm{J}}\left(1-\frac{r_{m J}}{r}\right)
\end{array}\right. \\
& \left\{\begin{array}{l}
b_{\mathrm{J}}=\frac{\left(a_{\mathrm{F}}-a_{\mathrm{V}}\right) b_{\mathrm{F}} r_{\mathrm{F}}^{2}}{b_{\mathrm{F}} r_{\mathrm{F}}^{2}+b_{\mathrm{F}} r_{\mathrm{J}}^{2}-a_{\mathrm{F}} r_{\mathrm{J}}^{2}+a_{\mathrm{V}} r_{\mathrm{J}}^{2}-2 b_{\mathrm{F}} r_{\mathrm{J}} r_{\mathrm{F}}} \\
r_{\mathrm{M}}=\frac{b_{\mathrm{J}} r_{\mathrm{J}}^{2}+b_{\mathrm{F}} r_{\mathrm{F}}^{2}}{b_{\mathrm{J}} r_{\mathrm{J}}+b_{\mathrm{F}} r_{\mathrm{F}}}
\end{array}\right.
\end{aligned}
$$

\section{Regional reconstruction technology for the iono- sphere parameters}

The transmitter is located in Wuhan, and the receiver is located in Beijing. Therefore, we could obtain vertical sounding data of the two places only. We employ regional reconstruction technology for the ionosphere parameters to determine the parameters of various layers in the midpoint along the great circle path. The method is described below [Liu (W.) et al. 2008].

Firstly, ionosphere distance $d_{i j}$ is computed by using Equation (11), in which Lon ${ }_{i}$ and Lat ${ }_{i}$ represent the longitude and latitude of the $i$ th place, respectively; the unit is degree; and $i=1,2 ; j=1,2$. The parameter SF refers to the scalar factor. The value of SF is 2 in the middle latitude area. 


$$
d_{i j}=\sqrt{\left(\operatorname{Lon}_{i}-\operatorname{Lon}_{j}\right)^{2}+S F\left(\operatorname{Lat}_{i}-\mathrm{Lat}_{j}\right)^{2}}
$$

Secondly, weight coefficients W1 and W2 are computed with Equations (12a) and (12b), in which $\mu$ denotes the Lagrange multipliers, and $d_{i 0}$ is the ionosphere distance between the $i$ th place and the midpoint.

$$
\left\{\begin{array}{l}
\sum_{j=1}^{2} d_{i j} \mathrm{~W}_{j}+\mu=d_{i 0} \quad i=1,2 \\
\sum_{j=1}^{2} \mathrm{~W}_{j}=1
\end{array}\right.
$$

Lastly, the parameters in the midpoint are estimated with the computed weight coefficients and the known parameters of the transmitting and receiving regions. For the parameters that could be obtained from IRI2012, such as the peak heights and the critical frequencies of different layers, we utilise Equation (13) as follows:

$$
\frac{z_{0}-\bar{z}_{0}}{\bar{z}_{0}}=\sum_{j=1}^{2} \mathrm{~W}_{j} \frac{z_{j}-\bar{z}_{j}}{\bar{z}_{j}}
$$

The parameters of IRI2012 are used as the background to retain the regional character of the ionosphere and to improve the accuracy of the reconstructed parameters, where $z_{0}$ is the estimated parameter, and $\bar{z}_{j} j=0,1,2$ is the counterpart to parameter $z_{j}$ from IRI2012. For the parameters which cannot be found in IRI2012, we apply Equation (14).

$$
z_{0}=\sum_{j=1}^{2} \mathrm{~W}_{j} z_{j}
$$

\section{Experiments}

Three oblique sounding ionograms between Wuhan and Beijing are selected. The times at which the data were collected are listed as follows: January 3, 2014, at 16:22 (UT), January 4, 2014, at 03:07 (UT) and May 25, 2014, at 02:07 (UT), which correspond to the nighttime scenario without the F1 layer, the daytime scenario without the F1 layer and the daytime scenario with the F1 layer, respectively. The one-hop oblique sounding ionograms generated with the three MQP models are compared with the experimental oblique sounding ionogram, as depicted in Figures 3, 4, and 5. The group path of the one-hop E layer is unaffected by

\begin{tabular}{|c|c|c|c|c|c|c|c|c|c|c|c|c|}
\hline $\mathrm{f}(\mathrm{MHz})$ & 2 & 2.2 & 2.4 & 2.6 & 2.8 & 3.0 & 3.2 & 3.4 & 3.6 & 3.8 & 4.0 & 4.2 \\
\hline $\mathrm{P}_{\exp }-\mathrm{P}_{\mathrm{MQP}-\mathrm{V}}(\mathrm{km})$ & -10 & -14 & -13 & -21 & -23 & -26 & -28 & -24 & -24 & -24 & -33 & -110 \\
\hline $\mathrm{P}_{\exp }-\mathrm{P}_{\mathrm{MQP}-\mathrm{VD}}(\mathrm{km})$ & -79 & -75 & -65 & -64 & -56 & -46 & -35 & -16 & -1 & 16 & 25 & -34 \\
\hline $\mathrm{P}_{\exp }-\mathrm{P}_{\mathrm{MQP}}(\mathrm{km})$ & -93 & -89 & -78 & -75 & -66 & -55 & -40 & -19 & 0 & 21 & 35 & -19 \\
\hline
\end{tabular}
the EF valley; therefore, the E layer group paths are not

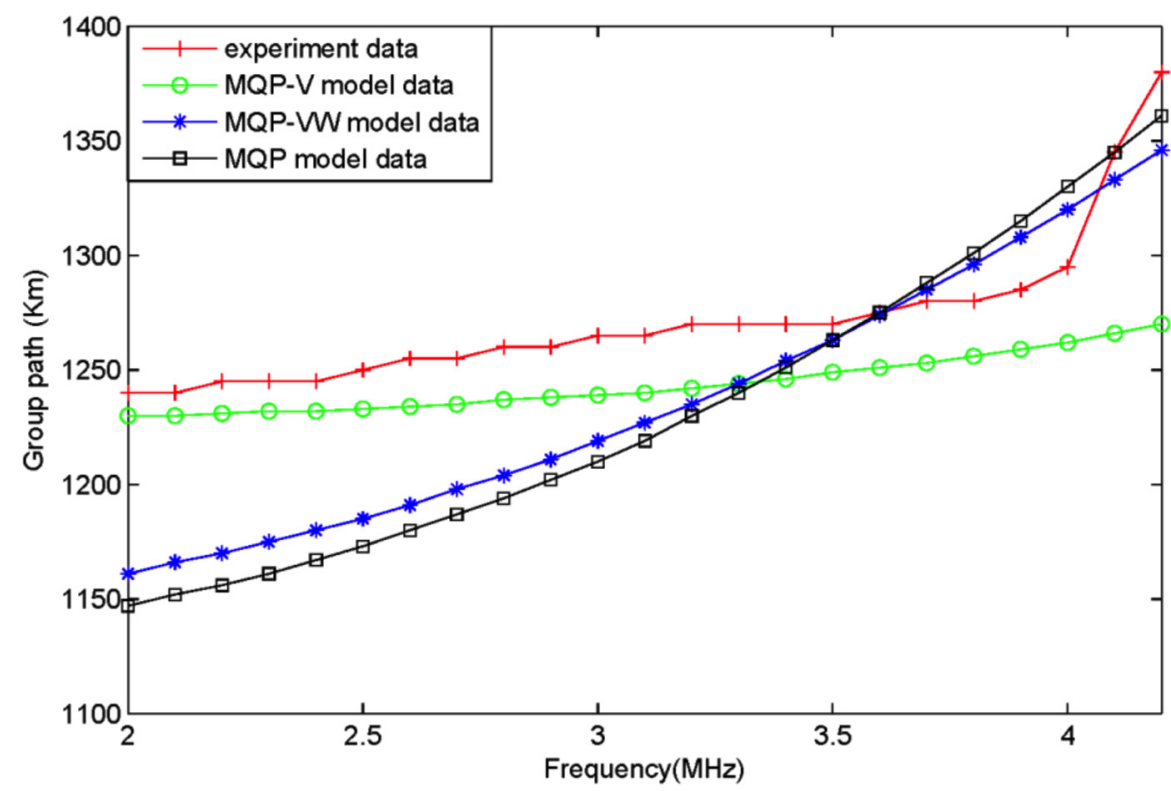

Figure 3. One-hop oblique sounding ionograms generated with the three MQP models and the experimental ionogram. Time: January 3, 2014, at 16:22 (UT).

Table 1. Difference values in the one-hop group paths of the experimental oblique sounding and of the simulation results. Time: January 3, 2014, at 16:22 (UT). 
displayed in the figures. The corresponding difference values in the one-hop group paths of the experimental oblique sounding and of the simulation results of the three MQP models are listed in Tables 1, 2 and 3, respectively. $\mathrm{P}_{\exp }-\mathrm{P}_{\mathrm{MQP}-\mathrm{V}}$ represents the one-hop group path of the experimental oblique sounding minus onehop group path of the MQP-V model, $\mathrm{P}_{\exp }-\mathrm{P}_{\mathrm{MQP}-\mathrm{VD}}$ represents the one-hop group path of the experimental oblique sounding minus the one-hop group path of MQP-VD model, $\mathrm{P}_{\exp }-\mathrm{P}_{\mathrm{MQP}}$ represents the one-hop group path of the experimental oblique sounding minus the one-hop group path of the MQP model.

Figure 3 and Table 1 illustrate that the oblique sounding ionogram generated by using the MQP-V model is similar to the experimental sounding ionogram and that the group path error is small, except for high frequencies close to MUF. Meanwhile, the ionograms generated with MQP-VW and the conventional MQP are less similar to the measured ionogram, and the group paths of the former change rapidly, but those of the latter shift gradually. The group path error observed at low frequencies is greater than $50 \mathrm{~km}$; nonetheless, the ionogram generated with the MQP-VW model is slightly superior to that which was generated by using the conventional MQP model. Abrupt changes do not occur in the ionograms generated with the three MQP models, unlike in the measured ionogram near MUF, because the vertical data utilised to obtain the parameters of various layers are not highly accurate.

Figure 4 and Table 2 illustrate that the oblique sounding ionogram generated by employing the MQP$\mathrm{V}$ model is highly similar to that of the measured ionogram. The group path error is small, and the changes at MUF are also similar. Meanwhile, the ionograms generated with MQP-VW and the conventional MQP are less similar to the experimental ionogram; the group paths change evidently with frequency, and the variations in MUFs are greater than those in its experimental counterpart. The MUF of MQP-V is $0.3 \mathrm{MHz}$ less than that of the experimental counterpart, whereas the MUFs of MQP-VW and MQP are $1.3 \mathrm{MHz}$ less than that of the experimental ionogram. The errors observed at low frequencies of MQP-VW and MQP are large, and the maximum errors at $10 \mathrm{MHz}$ are 40 and $44 \mathrm{~km}$ for MQPVW and MQP, respectively. Nonetheless, the oblique sounding ionogram generated with the MQP-VW

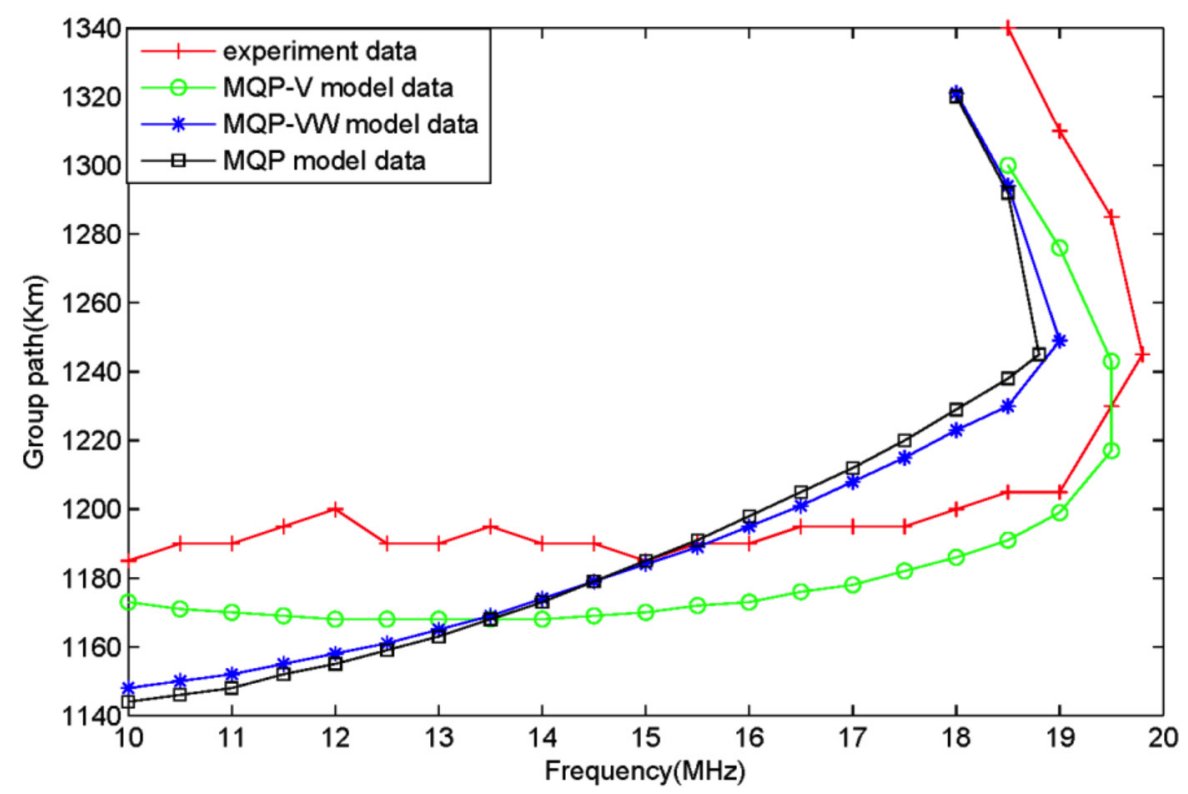

Figure 4. One-hop oblique sounding ionograms generated with the three MQP models and the experimental ionogram. Time: January 4, 2014, at 03:07 (UT).

\begin{tabular}{|c|c|c|c|c|c|c|c|c|c|c|c|}
\hline \multirow[t]{2}{*}{$\mathrm{f}(\mathrm{MHz})$} & \multicolumn{10}{|c|}{ F2 at a low angle } & \multirow{2}{*}{$\frac{\mathrm{F} 2 \text { at a high angle }}{18.5}$} \\
\hline & 10.5 & 11.5 & 12.5 & 13.5 & 14.5 & 15.5 & 16.5 & 17.5 & 18.5 & 19.5 & \\
\hline $\mathrm{P}_{\exp }-\mathrm{P}_{\mathrm{MQP}-\mathrm{V}}(\mathrm{km})$ & -19 & -26 & -22 & -27 & -21 & -18 & -19 & -13 & -14 & -13 & -40 \\
\hline $\mathrm{P}_{\exp }-\mathrm{P}_{\mathrm{MQP}-\mathrm{VD}}(\mathrm{km})$ & -40 & -40 & -29 & -26 & -11 & -1 & 6 & 20 & 25 & ---- & -46 \\
\hline$P_{\exp }-P_{M Q P}(k m)$ & -44 & -43 & -31 & -27 & -11 & 1 & 10 & 25 & 33 & ---- & -48 \\
\hline
\end{tabular}

Table 2. Difference values in the one-hop group paths of the experimental oblique sounding and of the simulation results. Time: January 4, 2014, at 03:07 (UT). 


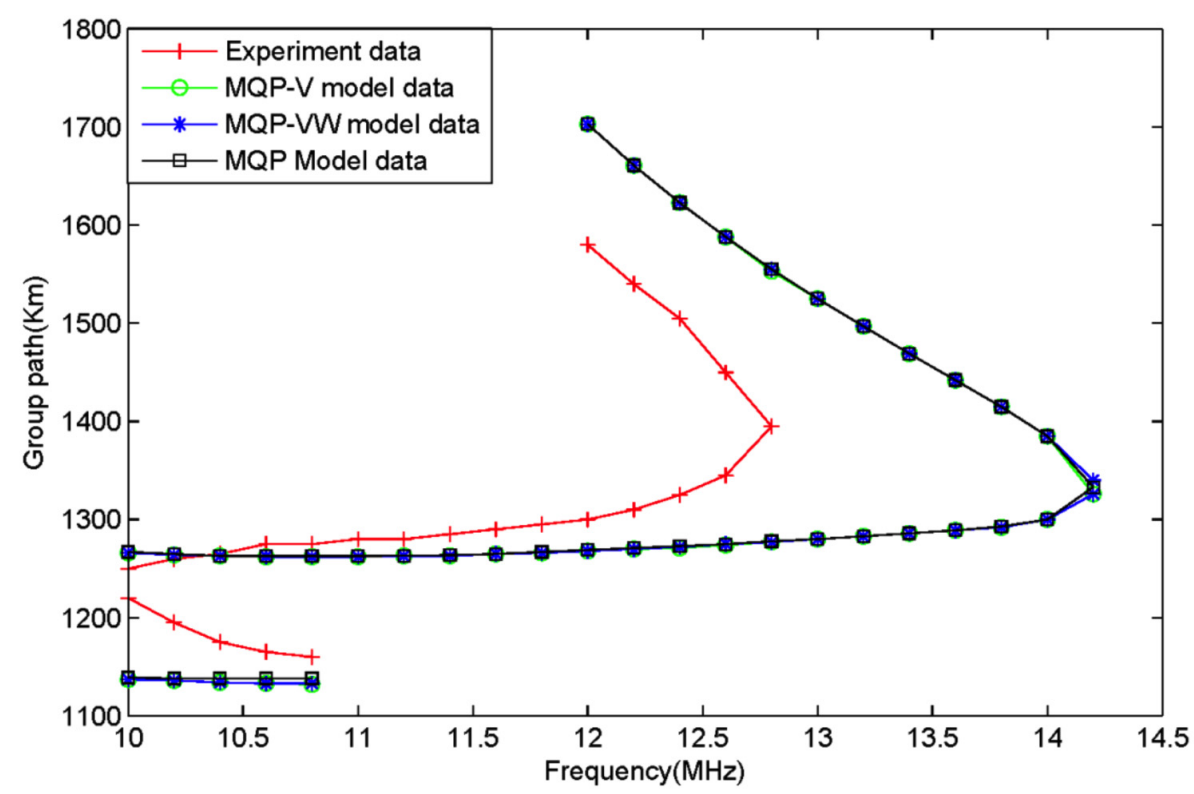

Figure 5. One-hop oblique sounding ionograms generated with the three MQP models and the experimental ionogram. Time: May 25, 2014, at 02:07 (UT).

\begin{tabular}{|c|c|c|c|c|c|c|c|c|c|c|}
\hline \multirow[t]{2}{*}{$\mathrm{f}(\mathrm{MHz})$} & \multicolumn{2}{|c|}{$\mathrm{F} 1$} & \multicolumn{5}{|c|}{ F2 at a low angle } & \multicolumn{3}{|c|}{ F2 at a high angle } \\
\hline & 10 & 10.8 & 10 & 10.8 & 11.6 & 12.4 & 12.8 & 12 & 12.4 & 12.8 \\
\hline $\mathrm{P}_{\exp }-\mathrm{P}_{\mathrm{MQP}-\mathrm{V}}(\mathrm{km})$ & -83 & -28 & 16 & -13 & -25 & -54 & -118 & 123 & 118 & 158 \\
\hline $\mathrm{P}_{\text {exp }}-\mathrm{P}_{\mathrm{MQP}-\mathrm{VD}}(\mathrm{km})$ & -83 & -27 & 16 & -13 & -25 & -53 & -118 & 123 & 118 & 160 \\
\hline$P_{\exp }-P_{M Q P}(k m)$ & -81 & 122 & 17 & -12 & -25 & -52 & -117 & 123 & 118 & 160 \\
\hline
\end{tabular}

Table 3. Difference values in the one-hop group paths of the experimental oblique sounding and of the simulation results. Time: May 25 , 2014, at 02:07 (UT).

model is slightly superior to the oblique ionogram generated with the conventional MQP model.

Figure 5 and Table 3 indicate that the oblique sounding ionograms generated with the three MQP models differ slightly from one another. This variation is attributed to the low predicted depth and width values of the EF-valley from IRI2012 in the daytime with F1, thereby leading to a shallow EF valley and a slight effect on radio propagation.

\section{Conclusion}

For the ionosphere in the daytime with the F1 layer, the oblique sounding ionograms generated by the three MQP models are less similar to the measured ionogram. By contrast, for the ionosphere without the F1 layer, the ionogram generated by using the MQP-V model is superior to those generated by using MQPVW and the conventional MQP model. The MQP-VD model maintains not only the valley depth parameter, but also the width of the valley bottom, whereas the MQP-VDW cannot guarantee the width of the valley bottom. This finding suggests that the depth and width of the valley bottom play an important role in electromagnetic propagation. Compared with the conven- tional MQP model, the MQP-V model is more suitable for describing the ionosphere without the F1 layer to increase the accuracy of ray tracing and for reducing the error of parameter inversion based on the ionosphere model.

Acknowledgements. This study was supported by the National Natural Science Foundation of China (grant no. 61172017).

\section{References}

Baker, D.C., and S. Lambert (1989). Range estimation for SSL HFDF systems by means of a multiquasiparabolic ionospheric model, IEE Proceedings $\mathrm{H}$ (Microwaves, Antennas and Propagation), 136 (2), 120-125.

Bourgeois, D., C. Morisseau and M. Flecheux (2005). Quasi-parabolic ionosphere modeling to track with Over-The-Horizon Radar. IEEE/SP Workshop on Statistical Signal Processing, 962-965.

Bourgeois, D., C. Morisseau and M. Flecheux (2006). Over-the-horizon radar target tracking using multiquasi-parabolic ionospheric modeling, IEE Proceedings-Radar, Sonar and Navigation, 153 (5), 409-416.

Dyson, P.L., and J.A. Bennett (1988). A model of the 
vertical distribution of the electron concentration in the iono-sphere and its application to oblique propagation studies, Journal of Atmospheric and Terrestrial Physics, 50 (3), 251-262.

Gasse, V., D. Lemur and L. Bertel (1999). A 3D ray tracing procedure to study ionospheric tilts, Physics and Chemistry of the Earth, Part C: Solar, Terrestrial \& Planetary Science, 24 (4), 379-383.

Hou, C.Y., G. Ke and Y. Fu (2015). The sky-wave radar detection performance computing based on the dynamic ionospheric model, Neurocomputing, 151, 1305-1315.

Krasheninnikov, I.V., J.C. Jodogne and L.F. Alberca (1996). Compatible analysis of vertical and oblique ionospheric sounding data, Annali di Geofisica, 39 (4), 763-768.

Li, W.M., D.L. Su, Z.W. Yang and Y. Liu (2012). Ionosphere hybrid modeling method for short-wave ray tracing, Journal of Beijing University of Aeronautics and Astronautics, 38 (4), 473-477.

Li, X.D., Q. He, B.X. Han and Z.S. He (2012). Detection Performance of MIMO-OTH Radar: Advantages of Multipath Ionospheric Propagation//Computer and Information Technology (CIT), 2012 IEEE 12th International Conference on IEEE, 758-762.

Liu, R.Y., G.H. Liu, J. Wu, B.C. Zhang, J.Y. Huang, H.Q. $\mathrm{Hu}$ and $\mathrm{Z} . \mathrm{H}$. $\mathrm{Xu}$ (2008). Ionospheric $\mathrm{f}_{\mathrm{O}} \mathrm{F}_{2}$ reconstruction and its application to the short-term forecasting in China region, Chinese Journal of Geophysics, 51 (2), 300-306.

Liu, W., P.N. Jiao, S.K. Wang and J.J. Wang (2008). Short wave ray tracing in the ionosphere and its application, Chinese Journal of Radio Science, 23 (1), 41-48.

Mahajan, K.K., R. Kohli, V.K. Pandey and N.K. Sethi (1990). Information about the E-region valley from incoherent scatter measurements, Advances in Apace Research, 10 (8), 17-20.

Wang, J., W. Fu, X.Z. Feng and D.M. Lu (2009). Technique on short distance high frequency single station location, Electronic Warfare Technology, 24 (1), 25-28.

Wang, S.K., W. Liu, S.L. Li, Y.B. Guo, J.M. Fan and P.N. Jiao (2010). Inversion method of F1 layer on vertical sounding ionogram, Chinese Journal of Radio Science, 25 (1), 172-177.

\footnotetext{
^Corresponding author: Xiao-Juan Zhang, Key Laboratory of Electromagnetic Radiation and Sensing Technology, Chinese Academy of Sciences, Beijing, China; email: xjzhang@mail.ie.ac.cn. 